\title{
FATORES INTERVENIENTES NO CUIDADO À PESSOA COM ÚLCERA VENOSA SOB A ÓTICA DE FAMILIARES
}

Saionara Leal Ferreira ${ }^{1}$

Islene Victor Barbosa ${ }^{1}$

Cecilia Freitas de Araújo Mota ${ }^{1}$

Solange Gurgel Alexandre ${ }^{2}$

Rita Neuma Dantas Cavalcante de Abreu ${ }^{1}$

Rita Mônica Borges Studart ${ }^{1}$
ORCID: https://orcid.org/0000-0002-4131-7077

ORCID: https://orcid.org/0000-0003-3523-7238

ORCID: https://orcid.org/0000-0002-9508-5312

ORCID: https://orcid.org/0000-0001-6742-1043

ORCID: https://orcid.org/0000-0002-5130-0703

ORCID: https://orcid.org/0000-0002-5862-5244

Objetivo: Identificar os saberes e as dificuldades encontradas por familiares no cuidado à pessoa com úlcera venosa no âmbito domiciliar Método: Estudo descritivo, de abordagem qualitativa, realizado em Fortaleza/CE, Brasil, em 2019, com oito familiares de pessoas com úlcera venosa, acompanhadas em ambulatório especializado de hospital público de ensino. Realizaram-se entrevistas semiestruturadas, cujas falas foram submetidas à análise de conteúdo temática proposta por Bardin. Resultados: A partir da análise das falas, surgiram três categorias temáticas: Saberes dos familiares no cuidado à úlcera venosa; Necessidade da orientação para manutenção do cuidado no domicílio; e Influência da úlcera venosa na dinâmica familiar. Conclusão: A identificação das lacunas no conhecimento dos entrevistados foi de grande relevância para o planejamento de tecnologia educativa voltada às necessidades encontradas, de maneira a oferecer suporte e alento a esse público.

Descritores: Úlcera Varicosa; Cuidados de Enfermagem; Familia; Processo de Enfermagem.

\section{INTERVENING FACTORS IN CARE OF PEOPLE WITH VENOUS ULCERA, UNDER THE FAMILY OPTICS}

Objective: To identify the knowledge and difficulties encountered by family members in caring for people with venous ulcers at home. Method: Descriptive, qualitative study, conducted in Fortaleza / CE, Brazil, in 2019, with eight relatives of people with venous ulcers, followed in a specialized outpatient clinic of a public teaching hospital. Semi-structured interviews were conducted, whose speeches were submitted to the thematic content analysis proposed by Bardin. Results: From the analysis of the speeches, three thematic categories emerged: Knowledge of family members in care for venous ulcer; Need for guidance to maintain care at home; and Influence of venous ulcer on family dynamics. Conclusion: The identification of gaps in the interviewees' knowledge was of great relevance for the planning of educational technology focused on the needs encountered, in order to provide support and encouragement to this audience.

Descriptors: Varicose Ulcer; Nursing Care; Family; Nursing Process.

\section{FACTORES INTERVENTIVOS EN CUIDADO DE PERSONAS CON ULCERA VENOSA, BAJO LA ÓPTICA FAMILIAR}

Objetivos: Identificar el conocimiento y las dificultades que enfrentan los miembros de la familia en el cuidado de las personas con úlceras venosas en el hogar. Metodo: Este es un estudio descriptivo y cualitativo realizado en Fortaleza / CE, Brasil, en 2019, con ocho familiares de personas con úlceras venosas, seguido en una clínica ambulatoria especializada de un hospital público de enseñanza. Se realizaron entrevistas semiestructuradas, cuyos discursos fueron sometidos al análisis de contenido temático propuesto por Bardin. Resultados: Del análisis de los discursos, surgieron tres categorías temáticas: conocimiento de los miembros de la familia en el cuidado de la úlcera venosa; Necesidad de orientación para mantener la atención en el hogar; e Influencia de la úlcera venosa en la dinámica familiar. Conclusión: La identificación de lagunas en el conocimiento de los entrevistados fue de gran relevancia para la planificación de la tecnología educativa centrada en las necesidades encontradas, con el fin de proporcionar apoyo y aliento a esta audiencia.

Descriptores: Úlcera varicosa; Atención de enfermeria; Familia; Proceso de enfermería.

${ }^{1}$ Universidade de Fortaleza - UNIFOR

${ }^{2}$ Hospital Universitário Walter Cantidio - HUWC

Autor correspondente: Saionara Leal Ferreira E-mail: enfernara@yahoo.com.br

Recebido: 21/06/2019

Aceito: 11/02/2020 


\section{INTRODUÇÃO}

A úlcera venosa tem como principal fator etiológico a insuficiência venosa crônica, constituindo ferida de difícil cicatrização e alta taxa de recidivas, necessitando de cuidados tópicos, uso da terapia compressiva para controle da hipertensão venosa e mudança no estilo de vida. O tratamento é longo e dispendioso, e as limitações que a ferida impõe contribuem para distanciar a pessoa acometida das atividades laborais e sociais, ainda em idade produtiva (1-2).

Os fatores que envolvem esse tipo de ferida, como o odor desagradável, o alto grau de exsudação, a dor, a mobilidade limitada e o isolamento social, afetam de forma negativa a qualidade de vida da pessoa, necessitando de cuidados no domicilio e acompanhamento em serviços de enfermagem especializados ${ }^{(3)}$.

Apesar da relevância do desenvolvimento da autonomia da pessoa com úlcera venosa para o cuidado de si ${ }^{(4)}$, a participação familiar nas ações inerentes ao cuidado pode repercutir na redução das recidivas. A assistência integral a essas pessoas ultrapassa o cuidado tópico, devendo - enfermeiro realizar ações de educação em saúde que integrem os familiares no processo terapêutico ${ }^{(5)}$.

Destarte, o enfermeiro deve pautar a atuação na Sistematização da Assistência de Enfermagem (SAE), elaborando e implementando intervenções, no intuito de identificar problemas, determinar metas, planejar intervenções e avaliar o cuidado. Tais ações elevam a qualidade da assistência e permitem a identificação precisa das necessidades do paciente e respectiva família(b), sendo os Diagnósticos de Enfermagem (DE) considerados imprescindiveis para direcionar a assistência de enfermagem (7).

Dentre os $\mathrm{DE}$ relacionados às pessoas com úlceras venosas, é importante destacar o "Controle ineficaz da saúde", que pode sinalizar a dificuldade na adesão ao tratamento prescrito, tendo como consequência o retardo na cicatrização e as recidivas da ferida. Este diagnóstico tem como fatores relacionados: apoio social insuficiente, conhecimento insuficiente do regime terapêutico e conflito familiar, e traz como intervenção a proposta do sistema de apoio familiar, capaz de melhorar as ações de cuidado e mobilizar essas pessoas ${ }^{(8)}$.

Assim, conhecer a realidade de familiares de pessoas em tratamento da úlcera venosa pode propiciar melhor condução no planejamento da assistência de enfermagem. de forma a integrá-los no processo terapêutico. Neste sentido, objetivou-se identificar os saberes e os fatores intervenientes encontrados por familiares no cuidado a pessoas com úlceras venosas no domicilio.

\section{METODOLOGIA \\ Tipo de estudo}

Trata-se de estudo descritivo, com abordagem qualitativa.

\section{Participantes da pesquisa}

A seleção da amostra deu-se por conveniência, sendo composta pelos familiares das pessoas com úlceras venosas de ambos os sexos. No primeiro atendimento a estas pessoas no serviço ambulatorial de estomaterapia, é realizada a Consulta de Enfermagem e deliberados os DE. A amostra do estudo foi composta pelos familiares de pacientes com o DE "Controle ineficaz da saúde".

Para sistematização da amostra, realizou-se análise prévia do agendamento do serviço para identificar as pessoas com o respectivo DE. Ao chegar ao serviço, o familiar era abordado pela pesquisadora que explicava os objetivos da pesquisa e o convidava a participar voluntariamente. No total, entrevistaram-se oito familiares, durante o mês de abril de 2019, sendo estabelecido o critério de saturação das informações para estimativa do número de participantes.

Os critérios de inclusão definidos foram: pessoas com 18 anos ou mais que moravam ou conviviam com a pessoa com a ferida, em pleno domínio de consciência. E os critérios de exclusão foram: pessoas que apresentassem estado de saúde físico ou mental comprometido, de modo a inviabilizar as respostas às perguntas da entrevista.

\section{Local de estudo}

O estudo foi realizado em ambulatório de atendimento especializado no cuidado a pessoas com feridas, em hospital Escola da Rede Pública, situado em Fortaleza, Ceará, Brasil.

\section{Coleta de dados}

Desenvolveu-se para o presente estudo instrumento com roteiro de entrevista semiestruturada, com questões abertas e fechadas, que abrangia a caracterização sociodemográfica dos participantes, conhecimentos sobre a úlcera venosa e as dificuldades e mudanças no planejamento de vida após o surgimento desta no familiar. A coleta das informações foi efetuada pela própria pesquisadora, a partir de entrevista individual, com a gravação de voz, após autorização dos sujeitos do estudo. Os participantes foram identificados pela letra $\mathrm{F}$ (Familiar), seguida do número indicativo da ordem da entrevista.

\section{Procedimentos de Análise de Dados}

Os dados coletados foram transcritos e analisadas qualitativamente, utilizando-se da técnica de Análise de Conteúdo que ocorre por meio da sistematização da descrição da mensagem recebida, buscando a sua compreensão(9), 
sendo agrupado em três categorias temáticas: Saberes dos familiares no cuidado à úlcera venosa; Necessidade da orientação para manutenção do cuidado no domicílio; e Influência da úlcera venosa na dinâmica familiar.

\section{Procedimentos Éticos}

O estudo foi aprovado pelo Comitê de Ética em Pesquisa, conforme parecer $n$ ㅇ 3.006.436 e n ․263.789. Os participantes assinaram o Termo de Consentimento Livre Esclarecido (TCLE), antes da entrevista.

\section{RESULTADOS}

Dos sujeitos do estudo, cinco eram do sexo feminino e três do masculino, estavam na faixa etária de 26 a 65 anos, com média de 39 anos, cinco eram casadas ou conviviam com companheiro (a), em regime de união estável, sendo dois solteiros e uma divorciada.

O tempo que o familiar auxiliava a pessoa no cuidado com a úlcera venosa variou de dois a 20 anos, coincidindo, na maioria dos casos, com o tempo em que a pessoa desenvolveu a ferida. O menor tempo de cuidado foi de nove anos e o maior de 20 anos, evidenciando a cronicidade da ferida.

$\mathrm{Na}$ análise dos dados obtidos na entrevista, destacaramse três categorias temáticas:

\section{Categoria 1: Saberes dos familiares no cuidado à úlcera venosa}

Evidenciou-se, pela fala dos entrevistados, fragilidade de compreensão acerca do que é a úlcera venosa, no entanto, a maioria relacionou a ocorrência da ferida a algum distúrbio do sistema venoso

Tenho pouco conhecimento, é um problema que dá na veia e, às vezes, abre a úlcera que é essas feridas que meu irmão tem (F3).

No caso da minha mãe, é uma úlcera devido ao excesso de peso dela e uma ferida demora a cicatrizar e dolorosa também e requer muito cuidado (F4)

Tenho nem ideia, acho que o motivo é a circulação dele (F8).

$\mathrm{Na}$ prática, enquanto enfermeira que atua no atendimento à pessoa acometida pela úlcera venosa, percebeu-se que o caráter crônico, devido ao tempo de convivência com o agravo, deveria contribuir para maior entendimento por parte dos familiares. Contudo, não se reconhece essa conotação, visto que estas pessoas podem se sentir desmotivadas pelo longo tempo dedicado ao cuidado à pessoa com a ferida.

Quando questionados sobre o motivo do retardo da cicatrização da ferida, alguns dos entrevistados afirmaram não saber o motivo, os demais citaram ausência de repouso, má circulação sanguínea, excesso de peso e ausência de bons hábitos alimentares.

A falta de entendimento do paciente em não ter repouso (F2).

Acho que é porque não circula direito o sangue (F3).

É o excesso do peso dela e a alimentação que não é tão saudáuel (F4).

Sei que demora, mas não sei o porquê (F7)

Para auxiliar uma pessoa com ferida crônica, é necessário que o familiar compreenda o processo que envolve o cuidado a ser dispensado fora do serviço de saúde, sendo importante a apropriação de informações básicas sobre a doença, a prevenção de condições crônicas e os cuidados com a ferida, além de buscar, observar e interagir com a equipe de saúde, durante o atendimento. Desta forma, o profissional de saúde que faz o acompanhamento, amplia o vínculo com o paciente e a família, o que poderá potencializar a continuidade do tratamento em âmbito domiciliar e, sobretudo, a melhoria da ferida

Quanto ao cuidado com a úlcera, questionou-se aos familiares sobre as dificuldades encontradas no espaço domiciliar, sendo citadas a não adesão ao repouso, questões de ordem econômica e conhecimento insuficiente acerca do tratamento.

Ele aceitar que para ela cicatrizar, precisa ter repouso absoluto (F2)

A questão financeira, nem sempre a gente tem o dinheiro para ela ter uma boa alimentação e para comprar os medicamentos e curativos (F5).

A demora que tem para cicatrizar, a úlcera dele fecha e depois abre novamente e ele fica impaciente (F8).

Nessa perspectiva, ao serem questionados sobre os cuidados que a pessoa deve ter, pontuaram-se o cuidado tópico com a ferida, o acompanhamento com o médico, a prática de atividade física, o repouso, a elevação do membro e o controle alimentar.

Repouso, faixa para melhorar a circulação e levantar as pernas. Ele passa o dia com as pernas levantadas (F3)

Não sei muito bem, sei que tem que ter o repouso (F5).

Higiene, lavar sempre o local, estar coberto com o curativo, evitar vários tipos de alimento para não estar inflamando (F6). 
Os entrevistados demonstraram conhecer os cuidados mais importantes para o processo cicatricial da úlcera venosa, necessitando, contudo, de melhor direcionamento e maiores estímulos para que tais ações façam parte da rotina diária da pessoa acometida.

\section{Categoria 2: Necessidade da orientação para manutenção do cuidado no domicílio}

Os familiares foram unânimes ao serem questionados sobre a necessidade de ter acesso ao material educativo que forneça informações sobre a úlcera venosa, expressando a importância deste no cotidiano de cuidados e o benefício esperado.

Ajudaria bastante, pois seria uma forma de ter conhecimento (...) quanto maior o conhecimento eu tiver para ajudar em casa, melhor será (FI).

É sim, toda a informação é boa, eu gostaria que tivesse uma cartilha para me orientar melhor (F3)

Bastante, porque têm coisas que eu nem sei e nem tudo é explicado, tem hora que a ferida está boa e tem hora que piora e eu que fico próxima a ela, observo bastante (F4).

Após avaliar as respostas, perceberam-se a angústia causada pelas dúvidas relacionadas ao tratamento e o sentimento de impotência e ansiedade, que podem ser minimizados pelo acesso a material educativo permanente que pode ser consultado em momento oportuno.

\section{Categoria 3: Influência da úlcera venosa na dinâmica familiar}

No momento da entrevista, ao serem questionados sobre as mudanças pessoais nos projetos de vida, após o surgimento da úlcera venosa no familiar, os participantes relataram, sobretudo, a necessidade de abandonar emprego ou manterse desempregado, assim como as mudanças nas atividades de lazer, devido à necessidade de dedicar-se à pessoa no auxílio aos cuidados diários, em especial com a ferida.

Já precisei sair de um emprego, pois minhas irmãs eram casadas e não podiam largar as famílias para ajudar no cuidado dela (FI)

Mudou, porque eu queria trabalhar, mas sou obrigada a fazer o curativo dele todos os dias, porque ninguém quer fazer (F3)

Mudou bastante porque eu tive que abrir mão de muita coisa por ela, porque ela não pode ficar sem uma pessoa para auxiliá-la (F4).

$\mathrm{Na}$ fala dos familiares, identificou-se a repercussão econômica que a ferida pode trazer, considerando que a pessoa acometida dificilmente manterá as atividades laborais Ainda sobre as alterações na vida daqueles que cuidam, questionou-se se havia ou houve variações nas condições fisiológicas e sociais, a exemplo do sono, descanso, lazer e participação social.

Atrapalha. Tanto eu como minha irmã, nós não podemos deixá-lo sozinho, não posso arranjar um emprego fixo (F2).

Quando a ferida está mais extensa, aumenta a preocupação (F3)

Não, eu o ajudo com maior satisfação, minha família é tudo que eu tenho (F7).

Os familiares envolvidos no processo terapêutico, também, sentirão o efeito de algumas das limitações impostas pelo adoecimento, já que, por questões afetivas ou solidárias, não mantêm as atividades que antes faziam parte da rotina.

\section{DISCUSSÃO}

$\mathrm{Na}$ assistência às pessoas com feridas crônicas, identificar os pontos que podem influenciar nos saberes e nas práticas dos familiares no domicílio, pode ser vista como ação colaborativa.

Entende-se que a incompreensão para realizar as práticas comunitárias de cuidado a serem desenvolvidos pela família poderá influenciar na adesão ao tratamento. Pois, conhecer as características das pessoas, para que as intervenções sejam planejadas e direcionadas a cada caso, pode ser vista como condição relevante na continuidade do tratamento ${ }^{(10)} \mathrm{e}$ melhoria da qualidade de vida das pessoas.

Conforme os achados do estudo, a presença maior da figura feminina no cuidado é corroborada pela literatura, quando se afirma que, no ambiente domiciliar, esta ação é, geralmente, exercida por cônjuges e filhos, em especial por mulheres. A satisfação do cuidar pode estar relacionada ao prazer e à valorização da pessoa cuidada, na medida em que essas pessoas se sentem retribuindo algo que receberam ${ }^{(11)}$.

Em relação ao conhecimento sobre a doença, estudo realizado com pessoas com úlcera venosa encontrou resultados semelhantes quanto à fragilidade do conhecimento das causas e dos cuidados que permeiam esta ferida(12) sendo o manejo desta desafiador, tanto para as pessoas que convivem com ela, quanto para o enfermeiro que implementa o tratamento tópico, essencialmente pelo caráter crônico e recorrente. Estudo de coorte acompanhou 50 pacientes adultos após cicatrização da úlcera venosa, durante 10 anos, mostrando que $62 \%$ apresentaram recidivas das feridas ${ }^{(2,13)}$.

A atenção a essas pessoas deve se concentrar em medidas que estimulem o retorno venoso, o controle da hipertensão venosa e o cuidado tópico com a ferida, de forma a promover 
ambiente adequado para que ocorra a cicatrização. A adesão a novos comportamentos deve fazer parte da rotina diária da pessoa com uso contínuo de terapia compressiva, a alternância dos momentos de repouso com práticas de caminhadas leves e o controle do peso corporal ${ }^{(13)}$

Ao ponderar a complexidade e a importância da adesão ao tratamento da úlcera venosa, a família é vista como fundamental suporte para o enfrentamento desta condição, na medida em que se configura como alicerce à pessoa doente, contribuindo para construção de um caminho de cuidados menos dolorosos. Os familiares são importantes atores a serem integrados ao cuidado terapêutico, especialmente por manter convívio diário e conhecer de perto as angústias e dificuldades que a pessoa com a ferida crônica possui ${ }^{(14)}$.

Para tal, o olhar diferenciado do profissional que assiste, por se tratar de cuidado contínuo, que deve ser adotado ao longo da vida e que traz em si alterações e adaptações para planejamentos futuros. Essas ações compartilhadas demandam o diálogo frequente e aberto, sendo condutos para se construir e implementar o plano terapêutico (15-16).

Conforme identificado nas falas dos familiares, a questão econômica é considerada fator agravante, pois a pessoa doente necessita de cuidados diários e contínuos, o que demanda recursos de diversas ordens e o orçamento familiar. Em situação econômica limitada, a presença da ferida e a atenção demandada são elementos capazes de desequilibrar o controle financeiro e emocional da família, afetando, assim, a qualidade de vida dos envolvidos ${ }^{(5)}$.

Estudo realizado em Petrópolis sinalizou o grau de limitações que a úlcera traz em relação à capacidade funcional, com a execução das atividades referentes ao equilibrio e à marcha, sendo consideradas com dependência parcial e o risco de quedas aumentado, o que demanda cuidados contínuos, sobretudo dos familiares ${ }^{(17)}$

Com base nos achados deste estudo, sugere-se que a Enfermagem faça uso de diversas estratégias para promoção da saúde, de forma a estimular o autocuidado, inserir os familiares no projeto terapêutico instituído, buscando prevenir as complicações da úlcera venosa e contribuir na recuperação e reabilitação da pessoa, para que seja mantido maior grau de independência no autocuidado, partindo da premissa de que a negligência destes fatores acarretará maior necessidade do cuidado assistido por familiares ${ }^{(17)}$.

Diante disso, na busca da efetivação do cuidado integral, o enfermeiro deve estabelecer relacionamento interpessoal e terapêutico com o público que deseja atingir, em busca da promoção de intervenções eficazes e resolutivas, considerando que, ao expandir o foco do cuidado clínico de enfermagem, consegue-se alcançar, em totalidade, a pessoa com a doença ${ }^{(18)}$, sendo importante pontuar que o uso das tecnologias educativas em saúde são importantes aliadas a este processo, na medida em que facilita o acesso da informação em tempo oportuno ${ }^{(19)}$.

\section{Limitações do estudo}

Aponta-se como limitação deste estudo o fato de ter sido desenvolvido em ambulatório especializado, refletindo realidade local, sendo relevante a execução de estudos em outros serviços, abrangendo esferas maiores.

\section{Contribuição do estudo para a prática}

Ofertar subsídios teóricos baseados em evidência para prática do cuidado de enfermagem em feridas, dando ênfase a importância da integração familiar no processo de cuidar e conviver com a úlcera venosa.

\section{CONCLUSÃO}

Constatou-se que os entrevistados tinham visão geral de que a úlcera venosa decorre de problemas relacionados ao sistema circulatório, percebiam as necessidades de cuidados importantes no tratamento, sendo estes, no entanto, práticas negligenciadas, fato que pode estar relacionado à ausência de compreensão sobre a sua importância.

Dentre as dificuldades encontradas, percebeu-se a influência de algumas das limitações impostas pela úlcera venosa, uma vez que, por questões afetivas ou solidárias, os familiares não mantêm as atividades que antes faziam parte da rotina, afetando a qualidade de vida destes. Assim, observaram-se lacunas quanto ao conhecimento dos entrevistados e fatores que intervém no cuidado à pessoa com ferida.

Desta forma, assevera-se a atuação de enfermeiros na disseminação de informações e no processo de educação em saúde para alcançar pessoas acometidas pela úlcera venosa, assim como familiares que fazem parte do convívio diário, considerando-os potenciais aliados na prática do cuidado que deve ser mantido no domicílio.

Agradecimentos: Ao Cofen e à CAPES, pela parceria firmada na fomentação de bolsas em mestrados profissionais voltadas aos enfermeiros.

Contribuições dos autores: Concepção, desenho, análise, interpretação dos dados e redação do artigo: Saionara Leal Ferreira, Islene Victor Barbosa, Cecilia Freitas de Araújo Mota, Solange Gurgel Alexandre, Rita Mônica Borges Studart. Revisão crítica e revisão final: Rita Neuma Dantas Cavalcante de Abreu. 


\section{REFERÊNCIAS}

1. Harding K, Dowsett C, Fias L, Jelnes R, Mosti G, Öien R, et al. Simplifying venous leg ulcer management. Consensus recommendations [Internet]. London: Wounds International; 2015 [acesso em 20 fev 2019]. Disponivel em: www.woundsinternational.com

2. Borges EL, Ferraz AF, Carvalho DV, Matos SS, Lima VLAN. Prevenção de recidiva de úlcera varicosa: um estudo de coorte. Acta Paul Enferm [Internet]. 2016 [acesso 29 nov 2019] ;29(1):9-16. Disponivel em: http://www.scielo.br/scielo.php?script=sci_arttext\&pid=S0103210020160001000098lng=en

3. Franks PJ, Barker J, Collier M, Gethin G, Haesler E, Jawien A, et al. Management of Patients With Venous Leg Ulcers: Challenges and Current Best Practice. J Wound Care [Internet]. 2016 [acesso em 20 fev 2019]; 25(Sup6): S1-S67. Disponivel em: http://www.magonlinelibrary.com/pb-assets/JOWC/EWMAvenous-leg-ulcers. pdf.

4. Finlayson K, Wu ML, Edwards HE. Identifying risk factors and protective factors for venous leg ulcer recurrence using a theoretical approach: a longitudinal study. Int J Nurs Stud [Internet]. 2015 [acesso em 20 fev 2019]; 52(6):1042-51. Disponivel em: https://www.ncbi.nlm.nih.gov/pubmed/25801312.

5. Brito DT, Xavier VMA, Santos JP, Agra G. Dor em úlcera crônica: perfil sociodemográfico, clínico e terapêutico de pacientes do municipio de Cuité - PB. Academus Rev Científica Saúde [Internet]. 2017 [acesso em 20 fev 2019]. 2(2). Disponivel em: https://smsrio. org/revista/index.php/reva/article/view/317.

6. Sousa HF, Araújo AM, Rosso ICASN, Freitas e Silva KR. O enfermeiro no manejo clínico de pacientes com úlcera venosa: revisão integrativa de literatura. Rev Humano Ser - UNIFACEX. 2015 [acesso em 20 fev 2019]; 1(1):32-51. Disponivel em: https://periodicos.unifacex.com.br/humanoser/article/view/623.

7. Channes M. SAE- Sistematização da Assistência de Enfermagem Descomplicada. São Paulo: Guanabara Koogan; 2018

8. Herdman TH, Kamitsuru S, NANDA International Inc. Diagnósticos de enfermagem da NANDA: definições e classificações 20182020. Porto Alegre: Artmed; 2018.

9. Bardin L. Análise de conteúdo. Lisboa: Edições 70; 2011.

10. Robaina ML, Budó MLD, Silva DC, Schimith MD, Durgante VL, Simon BS. Saberes e práticas de pessoas com úlcera venosa em tratamento com bota de Unna. Rev Enferm UFSM [Internet]. 2016 [acesso em 20 fev 2019]; 6(3): 371-81. Disponivel em: <https://periodicos.ufsm.br/reufsm/article/view/21985>

11. Aires M, Pizzol FLFD, Mocellin D, Rosset I, Morais EP, Paskulin LMG. Cross-cultural adaptation of the Filial Res- ponsibility protocol for use in Brazil. Rev Bras Enferm [Internet]. 2017. [acesso em $20 \mathrm{fev}$ 2019]; 70(6): 1268-76. Disponivel em: http://www.scielo.br/scielo.php?script=sci_arttext\&pid=S003471672017000601268\&lng=en.

12. Silva AS, Rodrigues TS, Meneses SM, Vera SO, Silva LM, Oliveira $\mathrm{MCL}$ et al. O cotidiano de pacientes com úlcera venosa: uma revisão integrativa. Rev Eletr Acervo Saúde [Internet]. 2017 [acesso em 20 fev 2019]; 9:871-82. 10. Disponivel em: https:// www.acervosaude.com.br/doc/REASlll.pdf

13. Fletcher F, Atkin L, Dowsett C, Hopkins A, Tickle J, Worboys F, et al. Best practice statement: holistic management of venous leg ulceration [Internet]. London: Wounds UK; 2016 [acesso em 20 fev 2019]. Disponivel em: www.wounds-uk.com.

14. Silva A, Santos E, Oliveira I, Viegas L, Santos M, Silva V, Costa C, Bisagni C, Jorge K. As úlceras vasculogênicas na perspectiva do cuidado de enfermagem. Rev Presença [Internet]. 2017 [acesso em 20 fev 2019]; 3(9):71-85. Disponivel em: http://revistapresenca.celsolisboa.edu.br/index.php/numerohum/article/ view/115.

15. Ribeiro G, Cavalcante TB, Santos KCB, Feitosa AHC, Silva BRS, Santos GLS. Pacientes internados com feridas crônicas: um enfoque na qualidade de vida. Enferm Foco [Internet]. 2019 [acesso em 27 nov 2019]; 10(2). Disponivel em: http://revista.cofen.gov.br/ index.php/enfermagem/article/view/1740.

16. Santos RSS, Sardinha AHL. Qualidade de vida de pacientes com doença renal crônica. Enferm Foco [Internet]. 2018 [acesso em 6 jul 2019]; 2(9):61-6. Disponivel em: http://revista.cofen.gov. br/index.php/enfermagem/article/view/1078.

17. Santos LSF. Capacidade funcional de pacientes com úlceras venosas no município de Petrópolis/RJ [dissertação]. Fluminense (RJ): Mestrado Acadêmico em Ciências do Cuidado em Saúde, Universidade Federal Fluminense; 2016. Disponivel em: https:// app.uff.br/riuff/handle/1/2430.

18. Teixeira AK. Cuidado clínico de enfermagem à pessoa com úlcera venosa: fundamentação na Teoria de Imogene King [dissertação]. Fortaleza (CE): Mestrado acadêmico, Universidade Estadual do Ceará; 2016. Disponivel em: http://www.uece.br/cmacclis/dmdocuments/anne.pdf.

19. Benevides JL, Coutinho JFV, Pascoal LC, Joventino ES, Martins MC, Gubert FA, et al. Development and validation of educational technology for venous ulcer care. Rev Esc Enferm USP. [Internet]. 2016. [acesso em 27 nov 2019];50(2):309-16. Disponivel em: http://www.scielo.br/scielo.php?script=sci_arttext\&pi$d=S 008062342016000200309 \&$ lng $=e n$. 